\title{
Akteure und Akteurskonstellationen alter(n)sgerechter Arbeitspolitik
}

In den nächsten Jahren wird der demografische Wandel mit steigenden Altersdurchschnitten der Belegschaften und sich wandelnden Rahmenbedingungen am Arbeitsmarkt für die Betriebe zunehmend zu einem wichtigen Einflussfaktor. Die allgemeine Erwartung ist, dass die Betriebe hierauf mit neuen personal- und arbeitspolitischen Strategien reagieren, die es ihnen nicht nur erlauben, ihre älteren Beschäftigten besser einzusetzen, sondern auch den Beschäftigten einen längeren Verbleib in der Arbeit zu ermöglichen. Dem stehen in der betrieblichen Realität jedoch große Umsetzungsdefizite gegenüber. Der Artikel fragt vor diesem Hintergrund nach den betrieblichen Akteuren und den von ihnen verfolgten demografiepolitischen Strategien.

KLAUS-PETER BUSS, MARTIN KUHLMANN

\section{Einleitung}

Der Betrieb ist ein zentraler Ort des Alterns in der Arbeitsgesellschaft. Seine Bedeutung für die Gestaltung des demografischen Wandels wird in den kommenden Jahren durch Eintritt der Kohorte der geburtenstarken Jahrgänge in das letzte Drittel ihrer Erwerbsbiografie noch unterstrichen werden: Bis zum Ende des Jahrzehnts wird der Anteil der über 50-Jährigen an der Erwerbsbevölkerung auf über $40 \%$ ansteigen. Seit vielen Jahren ist diese Entwicklung Gegenstand gesellschaftlicher Debatten sowie sozial- und arbeitswissenschaftlicher Forschung (Frerichs 1998; Rothkirch 2000; Deutscher Bundestag 2002; Buck et al. 2002; Kocka/ Staudinger 2009). Ein wiederkehrender Befund dabei lautet, dass es sowohl in Bezug auf die Beschäftigung Älterer als auch auf die Bedingungen eines gesunden Alterns in der Arbeit in den Betrieben notwendig sein wird, in einer ganzen Reihe von Politikfeldern Gestaltungsmaßnahmen zu entwickeln und betriebliche Politiken zu verändern (Morschhäuser 2006; Kistler et al. 2006; Kistler 2008; Bögel/ Frerichs 2011). Das Spektrum reicht von personalpolitischen Themen (Nachwuchssicherung, Work-Life-Balance, Qualifizierung usw.) über die Notwendigkeit der Sensibilisierung von Führungskräften für den demografischen Wandel bis hin zu Fragen einer alter(n)sgerechten betrieblichen Arbeitspolitik ${ }^{1}$ - Handlungsfelder, die sich seit Jahren auch in den Empfehlungen und Gestaltungshinweisen der ver- schiedenen gesellschaftlichen Akteure wiederfinden (BMBF 2010; BMAS 2010; Bertelsmann Stiftung/BDA 2008; IG Metall 2012a; BKK 2007; IfaA 2009). Die mit dem demografischen Wandel einhergehenden betrieblichen Handlungsnotwendigkeiten sind in der öffentlichen Debatte also in hohem Maße präsent.

Während in der Demografieliteratur vielfach die Tendenz vorherrscht, auf einen insgesamt hohen betrieblichen Handlungsdruck zu schließen und generalisierende Maßnahmenkataloge zu formulieren, dominiert in Studien zur betrieblichen Ebene die Einschätzung, dass in der Fläche bislang noch keine weitreichenden Veränderungen zu verzeichnen sind. Eine ganze Reihe von Befragungen aus den letzten Jahren legt den Schluss nahe, dass die Umsetzung demografiebezogener Maßnahmen in den Betrieben bislang kaum vorangeschritten ist und zwischen den Betrieben zudem differiert (Bellmann et al. 2007; Towers Watson 2011; DGFP 2011; IG Metall 2012b). Umsetzungsdefizite bestehen

\footnotetext{
1 Neben den klassischen Themen Arbeitsorganisation und Arbeitseinsatz fassen wir unter der Überschrift Arbeitspolitik auch andere Felder betrieblicher Gestaltungspolitik wie Entgeltsysteme und Leistungspolitik, betriebsorganisatorische Strukturen oder betriebliche Steuerungs- und Koordinationsformen. Im Mittelpunkt unseres Beitrags stehen somit betriebliche Gestaltungskonzepte und nicht Fragen der gesellschaftlichen Regulierung oder staatlichen Handelns.
} 
insbesondere im Handlungsfeld der betrieblichen Arbeitspolitik bzw. im Themenfeld Arbeitsgestaltung (Bellmann/ Leber 2011; Freidank et al. 2011; Latniak et al. 2010). Die häufig diagnostizierte Diskrepanz zwischen der hohen gesellschaftspolitischen Präsenz des Themas und seiner bisher begrenzten praktischen Relevanz im betrieblichen Handeln scheint dabei selbst für größere Unternehmen zuzutreffen (Becker et al. 2006).

Empirisch lassen sich in den Betrieben sehr unterschiedliche Politiken beobachten, und die von den Beschäftigten artikulierten Probleme rund um das Thema ,Altern' unterscheiden sich nicht nur entlang von Tätigkeitsfeldern (vgl. hierzu Georg et al. 2005; Grewer et al. 2007), sondern auch entlang der von Betrieben verfolgten Politiken. Ob ,gesundes Arbeiten` bis zur Rente möglich ist oder nicht, hängt, wie wir argumentieren werden, ganz wesentlich von den betrieblichen Akteurskonstellationen und den von den Akteuren verfolgten Demografiepolitiken ab. Die sich in den Betrieben rund um das Thema ,Demo-

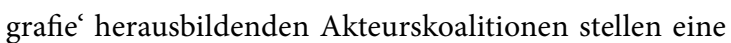
wichtige, in der Demografieliteratur vielfach jedoch unbeachtete Einflussgröße für betriebliche Demografiepolitik dar. Die von uns durchgeführten Fallstudien bestätigen insofern das generelle Bild, dass der demografische Wandel insbesondere von Personalbereichen und Betriebsräten zwar durchaus als relevantes Thema registriert wird, der Gestaltungsanspruch der Akteure und der Stand der Entwicklung und Umsetzung demografiepolitischer Konzepte insbesondere im Bereich einer alter(n)sgerechten Arbeitspolitik bislang jedoch noch vergleichsweise schwach ist. Die von uns in den Mittelpunkt gestellte Akteursperspektive zielt auf die betrieblichen Wirkmechanismen und eröffnet neue Antwortmöglichkeiten auf die Frage nach Wegen zum Abbau der vielfach beklagten Umsetzungsdefizite.

In einem ersten Schritt (Abschnitt 2) werden wir die wichtigsten Akteure demografieorientierter Politiken mit ihren Handlungsbedingungen und Handlungslogiken genauer vorstellen. Bereits auf dieser Ebene lassen sich zusätzliche Gründe für die demografiepolitische Entwicklung in den Betrieben sowie insbesondere für die begrenzte Umsetzungsdynamik einer alter(n)sgerechten Arbeitspolitik benennen. In einem zweiten Schritt (3) werden wir dann genauer auf die Bedeutung betrieblicher Akteurskoalitionen für die Umsetzung betrieblicher Demografiepolitiken eingehen. Entsprechende Gestaltungshinweise skizziert Abschnitt 4. Die empirische Basis unserer Argumentation bilden Befunde eines empirischen Forschungsprojektes „Problemlagen und Durchsetzungsbedingungen alter(n)sgerechter Arbeitspolitik“, in dem wir betriebliche Demografiepolitiken in verschiedenen industriellen Sektoren aus der Perspektive unterschiedlicher betrieblicher Akteure untersucht haben. Dieses Projekt wurde am Soziologischen Forschungsinstitut (SOFI) an der Universität Göttingen durchgeführt. ${ }^{2}$

\section{Betriebliche Akteure: Handlungs- bedingungen und Orientierungen}

\subsection{Demografischer Wandel als betriebliches Politikfeld}

Als Erklärung dafür, dass sich trotz großer öffentlicher Aufmerksamkeit für das Thema, demografischer Wandel' nach wie vor eine geringe Verbreitung alter(n)sgerechter Gestaltungsansätze beobachten lässt, lassen sich zunächst ein paar offensichtliche Gründe nennen: Erstens sind die Betriebe vom demografischen Wandel oftmals noch nicht in dem Maße betroffen wie dies der öffentliche Diskurs nahelegt. Mit dem bevorstehenden Eintritt der Kohorte der geburtenstarken Jahrgänge in die Altersphase, über 50` und der gleichzeitigen Anhebung des gesetzlichen Renteneintrittsalters dürften sich die Handlungsnotwendigkeiten in den Betrieben in den kommenden Jahren jedoch deutlich erhöhen. Zweitens variiert die Betroffenheit der Betriebe sowohl nach Tätigkeiten und Branchenbesonderheiten als auch in Abhängigkeit von der bislang betriebenen Personalpolitik erheblich. Gerade im industriellen Bereich hat eine Reihe von Betrieben den Anteil Älterer in den letzten Jahren durch Vorruhestandsregelungen und Altersteilzeit gesenkt. Primär personalpolitisch ausgerichtete Maßnahmen haben vielfach einen nach wie vor großen Stellenwert. Drittens sind gerade Arbeitsgestaltungsmaßnahmen oftmals mit Investitionen verbunden. Die Erträge dieser Investitionen sind i. d. R. weniger gut berechenbar und/oder stellen sich erst zu späteren Zeitpunkten ein. Der Hinweis auf Durchsetzungsschwierigkeiten, die aus dem Aufeinandertreffen unterschiedlicher Zeithorizonte von langfristig angelegten alter(n)sbezogenen Personal- und Arbeitspolitiken mit kurzfristig ausgerichteten Kostenkalkülen resultieren, findet sich in etlichen Untersuchungen (vgl. u. a. Freidank et al. 2011; Latniak et al. 2010; Kistler 2008).

Bereits diese kurze Aufzählung verweist darauf, dass die Umsetzung demografiebezogener Maßnahmen in ho-

\footnotetext{
2 Im Rahmen dieses durch die Hans-Böckler-Stiftung geförderten Projektes (Projektnr. 2010-381-3) haben wir in den Jahren 2011-2012 vergleichende Betriebsfallstudien in drei Chemie-, vier Pharma-, drei Automobilbau- sowie fünf Maschinenbaubetrieben durchgeführt. Die Untersuchung zielte auf die betrieblichen Handlungsanforderungen sowie die Problemwahrnehmungen, Handlungsstrategien und Politiken der verschiedenen betrieblichen Akteure (Betriebsräte, Bereichsmanagement, Personalmanagement) und gibt auf dieser Basis einen Überblick über typische Problemlagen und Arbeitspolitikstrategien. In fünf ausgewählten Betrieben wurden im Rahmen von Intensivfallstudien zusätzlich die Sichtweisen der Beschäftigten in für die Betriebe typischen Tätigkeitsbereichen untersucht. Insgesamt wurden rund 90 Expertengespräche und 60 Beschäftigteninterviews durchgeführt.
} 
Politikfeld

Personalpolitik

strategischer Fokus

demografiepolitischer

Ansatz

exemplarische

Politikkonzepte

\begin{tabular}{|c|c|c|}
\hline \multicolumn{2}{|c|}{$\begin{array}{l}\text { Optimierung der betrieblichen } \\
\text { Personalstruktur }\end{array}$} & $\begin{array}{l}\text { individuelle Betriebsbindung, } \\
\text { verhaltensbezogene Prävention }\end{array}$ \\
\hline $\begin{array}{l}\text { Anpassung der } \\
\text { betrieblichen } \\
\text { Alterstruktur }\end{array}$ & $\begin{array}{l}\text { externe Sicherung } \\
\text { des Arbeitskräfte- } \\
\text { bedarfs }\end{array}$ & $\begin{array}{l}\text { beschäftigtenbezogen: } \\
\text { Erhalt / Sicherung qualifizierter } \\
\text { Arbeitskraft }\end{array}$ \\
\hline $\begin{array}{l}\text { Ausbildung (über } \\
\text { Bedarf, Übernah- } \\
\text { meregelungen); } \\
\text { Ausstiegsrege- } \\
\text { lungen für Ältere } \\
\text { (Vorruhestand, } \\
\text { Altersteilzeit) }\end{array}$ & $\begin{array}{l}\text { Employer Branding; } \\
\text { Erschließung } \\
\text { neuer Erwerbs- } \\
\text { tätigengruppen; } \\
\text { Rekrutierung / Leih- } \\
\text { arbeit }\end{array}$ & $\begin{array}{l}\text { Vereinbarkeit Familie / Beruf; } \\
\text { Work-Life-Balance; } \\
\text { individuelle Arbeitszeitflexibilisierung; } \\
\text { Personalentwicklung; } \\
\text { individuelle Gesundheitsförderung } \\
\text { und Beratung; } \\
\text { Sportangebote; } \\
\text { Ernährungsangebote }\end{array}$ \\
\hline
\end{tabular}

Personalmanagement / Betriebsrat
Personalmanagement
Personalmanagement oder Personalmanagement / Betriebsrat
Arbeitspolitik

\begin{tabular}{|c|c|}
\hline $\begin{array}{l}\text { altersbezogen: } \\
\text { Beschäftigung Älterer } \\
\text { ermöglichen }\end{array}$ & $\begin{array}{l}\text { alternsbezogen: } \\
\text { proaktive } \\
\text { Arbeitsgestaltung }\end{array}$ \\
\hline $\begin{array}{l}\text { Arbeitsorganisation, } \\
\text { altersdiff. Leistungs- } \\
\text { politik; Reduzierung } \\
\text { Schichtarbeit; } \\
\text { Altersteilzeit/-freizeit; } \\
\text { Ergonomie; } \\
\text { Weiterbildung für } \\
\text { Ältere; } \\
\text { Ausweicharbeitsplätze }\end{array}$ & $\begin{array}{l}\text { Arbeitsorganisation; } \\
\text { Leistungspolitik; } \\
\text { gesundheitsverträgli- } \\
\text { chere Schichtmodelle; } \\
\text { Langzeitkonten; } \\
\text { Ergonomie(-checks); } \\
\text { Lebenslanges Lernen; } \\
\text { arbeitsbezogene Ge- } \\
\text { sundheitsförderung } \\
\text { (Gesundheitszirkel } \\
\text { etc.) }\end{array}$ \\
\hline
\end{tabular}

Linienmanagement / Betriebsrat / Personalmanagement werden hierbei einerseits verbesserte Beschäftigungsmöglichkeiten für Ältere sowie andererseits arbeitsgestalterische Maßnahmen, die die Chance erhöhen, ein , gesünderes Altern' in der Arbeit zu ermöglichen.

Die von uns unterschiedenen Strategien stehen nicht notwendig in Konkurrenz zueinander, sondern können sich auch ergänzen. Häufig belassen es Betriebe auch nicht bei Einzelmaßnahmen, sondern verfolgen einen Mix unterschiedlicher Politikkonzepte, dessen Zusammensetzung sich jedoch nicht allein als Reaktion auf spezifische betriebliche Problemlagen erklären lässt. Die unterschiedlichen demografiepolitischen Strategien verweisen vielmehr auf Spielräume bei der betrieblichen Gestaltung alters- und alternsbezogener Politiken und damit auf die Rolle und Bedeutung der betrieblichen Akteure. Wie wir im Folgenden an den drei wichtigsten Akteursgruppen zeigen werden, ist deren Einfluss auf die betriebliche Demografiepolitik freilich höchst unterschiedlich, und es bestehen typische Unterschiede zwischen den Perspektiven der Akteure auf das Demografiethema und den von ihnen verfolgten Strategien.

\subsection{Personalmanagement (Personalbereiche)}

te Fachkräfte eher intern abzusichern durch die Förderung der individuellen Betriebsbindung sowie Maßnahmen aus dem Bereich der verhaltensbezogenen Prävention. In ähnlicher Weise primär auf die vorhandene Belegschaft ausgerichtet, wenngleich mit völlig anderer Stoßrichtung, sind schließlich arbeitspolitisch orientierte Strategien einer verhältnisbezogenen Prävention, in denen die Arbeitssituationen der Beschäftigten und die betrieblichen Arbeitseinsatzbedingungen in den Vordergrund rücken. Angestrebt
Fragt man nach betrieblicher Demografiepolitik, geht der Blick zunächst zu den für Personalfragen zuständigen Personalbereichen. Die angesichts des längerfristig wirksamen demografischen Trends naheliegende Erwartung ist, dass das Personalmanagement in der Umsetzung demografiepolitischer Strategien im Betrieb eine führende Rolle einnimmt. Unterstrichen werden solche Erwartungen von der 
personalwirtschaftlichen Literatur. Der demografische Wandel wird dort bereits seit Jahren als prägender Megatrend betont. Die in seiner Folge zu erwartenden Verwerfungen am Arbeitsmarkt würden die Unternehmen in ganz neuer Weise zwingen, so das Argument, sich mit Personalfragen auch strategisch auseinanderzusetzen und daher, so die vielfache Hoffnung, auch die Rolle des Personalmanagements im Unternehmen stärken (siehe etwa: Boden 2005; Pack 2002).

Tatsächlich ist das Thema demografischer Wandel, wie unsere Fallstudien zeigen, in den Personalbereichen bereits seit einigen Jahren angekommen, dort auf der Diskursebene fest verankert, und eine Vielzahl von Betrieben weiß von demografiebezogenen Maßnahmen zu berichten. Hierbei spielen personalpolitische Maßnahmen eine große Rolle, sodass die Personalbereiche einerseits - und ganz in Einklang mit den Erwartungen der personalwirtschaftlichen Literatur - als die vermeintlich eigentlichen Akteure betrieblicher Demografiepolitik erscheinen. Auf der anderen Seite zeigt unsere Empirie ein äußerst heterogenes Bild gerade der Personalbereiche, bestehen doch zugleich deutliche Unterschiede in Bezug auf die Handlungsfähigkeit gerade dieses betrieblichen Akteurs.

In einem Teil der Unternehmen finden sich durchaus betriebliche Personalabteilungen, die in diesem Feld strategisch handeln. Hier geht die Thematisierung des demografischen Wandels sehr stark von den zuständigen Personalern aus, die in Kooperation mit anderen Akteuren am Standort - auch proaktiv, ohne akuten Handlungsdruck vielfältige demografiepolitische Initiativen von Altersstrukturanalysen der einzelnen Bereiche und Abteilungen über Gesundheitsmaßnahmen (Gesundheitschecks, Vorsorge, Beratung usw.) und Sportangeboten bis hin zu Programmen familienfreundlicher Arbeitsbedingungen und Serviceangeboten an die Beschäftigten (inklusive Wäschereiservice) anstoßen. Mitunter werden auch Maßnahmen einer altersund alternsgerechten Arbeitspolitik von diesen Personalbereichen aktiv und in Kooperation mit den jeweiligen Fachbereichen verfolgt. Eine der zentralen Leistungen liegt hier in einer frühzeitigen, breiten betrieblichen Bewusstseinsbildung, durch die das Thema fest in den Köpfen sowohl der Führungskräfte als auch der Beschäftigten verankert wird. Ein solches Positivbeispiel findet sich etwa in einem Chemiebetrieb unseres Samples, in dem dieser Prozess bereits sehr früh - noch vor der Festschreibung solcher Maßnahmen im Demografie-Tarifvertrag der Chemischen Industrie aus dem Jahr 2008 - einsetzt. Zum Erhebungszeitpunkt versuchte man hier in einem Pilotbereich gemeinsam mit den Beschäftigten in Workshops alter(n)sbezogene Probleme - auch in Bezug auf Arbeitsorganisation/-gestaltung - zu erheben und Lösungen zu finden.

Diesem Positivbeispiel stehen allerdings andere Fälle gegenüber, in denen von den Personalabteilungen kaum arbeitsbezogene Initiativen ausgehen. Ein Teil der betrieblichen Personalabteilungen scheint von den Herausforderungen des demografischen Wandels vielmehr schlicht überfordert. Dies hängt zum einen an fehlenden Gestaltungsmöglichkeiten. So sind beispielsweise in der Automobilindustrie geeignete Arbeitsplätze für Beschäftigte mit gesundheitlichen Einschränkungen vielfach seit Langem ausgelagert und daher an den Standorten rar. Entsprechend begrenzt stellen sich die personalpolitischen Reaktionsmöglichkeiten auf altersbedingte Einsatzeinschränkungen dar. Zum anderen und vor allem aber haben gerade die Personalbereiche in den Unternehmen oftmals eine eher schwache Position und erweisen sich gegenüber der Unternehmensleitung und den Bereichsleitungen als nicht sonderlich durchsetzungsfähig, wenn es um arbeitspolitische Gestaltungsmaßnahmen geht. Zudem haben die betrieblichen Personalbereiche in den letzten Jahren vielfach einen Restrukturierungsprozess durchlaufen, im Zuge dessen das betriebliche Personalmanagement zu einer internen Dienstleistungsfunktion umgestaltet und Teile der vormals betrieblichen Personalarbeit zentralen Personalbereichen bzw. standortübergreifenden Service-Centern zugeordnet wurden. Dieser Restrukturierungsprozess ging nicht selten mit einem Abbau betrieblicher Personalmanagementkapazitäten und -kompetenzen einher und begrenzt entsprechend die Handlungsfähigkeit der betrieblichen Personalbereiche. Vielfach sehen diese sich nun sowohl mit den vom Tagesgeschäft geprägten konkreten betrieblichen Anforderungen als auch mit den Umsetzungsanforderungen zentral formulierter personalpolitischer Programme konfrontiert, die allerdings nicht immer auf die betrieblichen Besonderheiten abgestimmt sind. Mit darüber hinausreichenden eigenen demografiepolitischen Initiativen wären diese Personalbereiche oft schon allein personell schlichtweg überfordert. Auch wenn nicht alle Unternehmen diesem Reorganisationstrend folgen, finden sich solche Entwicklungen in einer Reihe von Fallstudienbetrieben und werden von den lokalen Personalverantwortlichen, die hierin auch eine Aushöhlung ihrer beruflichen Kompetenzen sehen, heftig kritisiert.

Die demografiepolitischen Handlungsmöglichkeiten der Personalbereiche und ihre Rolle als demografiepolitischer Akteur sind vor diesem Hintergrund eher begrenzt. Auch wenn von den Personalern vor Ort Initiativen ausgehen, nehmen sie insbesondere auf die arbeitspolitische Entwicklung der Betriebe kaum Einfluss, die im Umgang mit alternden Belegschaften jedoch zunehmend an Bedeutung gewinnt. Bei der Gestaltung von Arbeits- und Organisationsstrukturen haben die Personalbereiche vieler Unternehmen an Entscheidungskompetenzen und Einflussmöglichkeiten eingebüßt, und es finden sich Klagen, dass das vorhandene Know-how immer weniger gefragt ist und zunehmend schwindet. Fragen der Arbeits- und Organisationsgestaltung wandern im Zuge der seit einigen Jahren forcierten Einführung von Produktionssystemen immer stärker in die Zuständigkeit von vorwiegend technisch orientierten und häufig zentralistisch ausgerichteten Planungsabteilungen. Stattdessen konzentrieren sich die von Personalmanagern ausgehenden demografiebezogenen Aktivitäten oftmals auf Maßnahmen aus dem Reper- 
toire klassischer Personalarbeit, die weniger auf die Zustimmung anderer Akteure angewiesen und daher leichter zu realisieren sind. Im Vordergrund stehen dabei Strategien, die über die Optimierung der betrieblichen Altersstruktur hinaus die Förderung der individuellen Betriebsbindung und die verhaltensbezogene Prävention in den Vordergrund stellen (Übersicht 1). Nicht zuletzt aufgrund ihres geringen arbeitspolitischen Einflusses droht so aber die Frage nach der Alter(n)sgerechtheit der betrieblichen Arbeitseinsatzstrategien von den Personalabteilungen ausgeklammert zu werden, und die Auseinandersetzung mit den Folgen einer wenig alter(n)sgerechten Arbeitsorganisation wird vermehrt in die individuelle Verantwortung der Beschäftigten verwiesen.

\subsection{Fachbereichs-/Linienmanagement}

Während der demografische Wandel aus der Perspektive des Personalmanagements einen wichtigen Megatrend darstellt, der wesentliche Bereiche der eigenen Arbeit beeinflusst, bleibt er für das Management der Produktions- und anderer Fachbereiche oft eine noch eher abstrakte Größe. „Demografischer Wandel?“, so ein Produktionsleiter im Expertengespräch, „davon lese ich in der Zeitung.“ Im Management der Fachbereiche stehen andere als demografiepolitische Aufgaben im Vordergrund. Am deutlichsten wird dies am Beispiel der Produktionsbereiche, die in hohem Maße an der Erfüllung ihrer operativen Funktionen gemessen und zumeist über kurzfristig ausgerichtete Kennzahlen und Zielvorgaben gesteuert werden. Mittel- bis langfristig wirksam werdende demografiebedingte Probleme liegen zumeist außerhalb ihres Handlungshorizonts: „Ich muss meinen Laden natürlich so fahren, dass er wirtschaftlich ist. Es mag sein, dass es Sinn macht, heute Leute auszubilden, weil ich sehe, in fünf oder zehn Jahren hast du da ein Loch. Aber so weit gucke ich nicht. Dafür bin ich zu sehr durchsatzgetrieben [...] Ich kann sagen, in zehn Jahren brauchen wir Leute und dafür jetzt ausbilden. Aber dafür muss ich die Leute jetzt bezahlen. Und das ist natürlich schwierig, wenn ich eine Zielvorgabe Produktivität habe.“ (Bereichsleiter Endmontage in einem Maschinenbaubetrieb)

Mit altersbezogenen Fragen sehen sich Fachbereichsleitungen vor allem dann konfrontiert, wenn es in ihren Bereichen akuten Handlungsbedarf etwa infolge eines besonders hohen Altersdurchschnitts oder altersbedingter Einsatzeinschränkungen gibt. Typische Problemlagen äußern sich hier zum einen in Klagen über die unzureichende Lernfähigkeit bzw. Lernwilligkeit von Teilen der alternden Belegschaft (die nicht selten mit betrieblichen Weiterbildungsstrategien korrespondieren, die gerade diese Beschäftigten über lange Jahre aus ihrem Adressatenkreis ausgeklammert haben), zum anderen in Klagen über eine altersbedingt abnehmende physische Leistungsfähigkeit und zunehmende gesundheitliche Probleme. „Ich sage nur: Rücken ohne Ende! [...] Wir hatten vor zehn Jahren vielleicht ein bis zwei Kollegen mit gesundheitlichen Einschrän- kungen. Heute habe ich mindestens zehn Kollegen, die ich kaum noch einsetzen kann." (Leiter Instandhaltungsbereich Automobilunternehmen)

Klagen wie diese verweisen vielfach auf Folgen einer Arbeitspolitik, die durch eine wenig alterns- und beschäftigtengerechte Arbeitsgestaltung das Entstehen altersbedingter Probleme befördert hat, mit deren Konsequenzen sich die Fachbereichsleiter nun konfrontiert sehen.

Trotzdem mangelt es den Fachbereichsverantwortlichen vielfach nicht an Problembewusstsein. Mögliche Ansatzpunkte im Umgang damit liegen für das Fachbereichsmanagement vor allem im Bereich der Arbeitspolitik (Übersicht 1). In den von uns untersuchten Betrieben finden sich durchaus Beispiele für arbeitsorganisatorische Initiativen der Fachbereichsleitungen (oder unter deren Beteiligung). Häufig werden Investitionen in eine ergonomische Verbesserung der Arbeitsplätze vorgenommen, teils werden die Beschäftigten aktiv in die Entwicklung und Umsetzung von Maßnahmen einer alter(n)sgerechten Arbeitsplatzgestaltung einbezogen oder alter(n)sgerechte Formen der Arbeitsorganisation angestrebt. Solche Initiativen sind dem Fachbereichsmanagement im Rahmen seiner unmittelbaren Verantwortung für die Arbeitsorganisation und den Arbeitseinsatz durchaus möglich. Sie finden zum Teil aber auch Grenzen in zentralen Vorgaben. Der Betriebsleiter eines Chemiebetriebes bringt es auf den Punkt: „Demografie kostet Geld. Und das muss die Firma irgendwann mal diskutieren, dass es das nicht umsonst gibt. [...] Noch mehr Hilfsmittel kann ich da nicht hinsetzen. Ich kann den Bildschirm größer machen, irgendwelche Hörhilfen zur Verfügung stellen. Da kann ich alles Mögliche machen, aber das ist nicht die Lösung. Da doktore ich nur an den Symptomen rum.“ (Betriebsleiter, Chemie-Betrieb)

Je höher die Produktivitätsvorgaben und Kosteneinsparungsziele und je enger die Investitionsspielräume des Fachbereichsmanagements vor Ort sind, desto enger sind auch die arbeitspolitischen Handlungsspielräume. So verweist der zitierte Betriebsleiter im Expertengespräch auf die nicht zuletzt infolge zentraler Vorgaben anwachsende - ungute Mischung zunehmend verdichteter Leistungsanforderungen, einer immer knapperen Personalbemessung und einer zugleich alternden Belegschaft, die die Ansätze einer innovativen Arbeitsorganisation vor Ort immer mehr konterkariert und in deren Folge zunehmend Probleme mit Leistungsanforderungen und Schichtarbeit auftreten.

\subsection{Betriebliche Interessenvertretung}

Die Betriebsräte als dritte zentrale Akteursgruppe betrieblicher Demografiepolitik zeichnen sich zumeist durch hohes Problembewusstsein aus. In der Regel selber aus der Produktion oder anderen Fachbereichen stammend, kennen sie nicht nur Arbeitssituationen und Arbeitsbelastungen der Beschäftigten vor Ort vielfach noch sehr genau. Sie sind auch in ihrer alltäglichen Arbeit sehr häufig mit Folgeproblemen wenig alternsgerechter Arbeit konfrontiert: sei es 
bei der Suche nach alternativen Verwendungsmöglichkeiten für Kollegen mit Einsatzeinschränkungen, sei es im Zusammenhang mit der individuellen Beratung bei der Beantragung von Altersteilzeit. Gleichwohl bleibt der Umgang der Betriebsräte mit dem Thema demografischer Wandel in den meisten Fällen eher reaktiv.

Dies wird bereits daran deutlich, dass demografiepolitische Themen in den Arbeitsstrukturen der Betriebsräte, d.h. bei der Systematik von Ausschüssen oder bei der Gestaltung von Projektgruppen, bisher kaum Spuren hinterlassen haben. Gerade arbeitspolitische Themen werden eher von besonders engagierten Einzelpersonen vorangetrieben, wobei es allerdings auch Gegenbeispiele arbeitspolitisch proaktiv agierender Betriebsräte gibt. Der bislang eher begrenzte Stellenwert von Arbeitspolitik ist insofern nicht verwunderlich, als sich die Interessenvertretungen in den Kernbranchen der deutschen Industrie seit einer ganzen Reihe von Jahren in Auseinandersetzungen und Verhandlungen um Restrukturierungsprogramme, Produktionsverlagerungen und drohenden Personalabbau befinden, bei denen es vor allem um Beschäftigungssicherung und $\mathrm{Zu}$ geständnisse an die Arbeitgeberseite geht. Auch dort, wo die Notwendigkeit einer intensiveren Beschäftigung mit dem Thema Arbeitspolitik gesehen wird, waren Aufmerksamkeit und Spielräume für Fragen der Gestaltung der Arbeit und des Arbeitsumfeldes daher gering. Ähnlich wie in den Personalbereichen liegt die demografiepolitische Perspektive somit auch bei vielen Betriebsräten stattdessen schwerpunktmäßig auf dem Bereich der Personalpolitik (Übersicht 1).

Zumeist bedeutet Demografiepolitik für Betriebsräte vor allem die Begleitung von oder aktive Mitarbeit bei Initiativen des Personalmanagements, in denen sie aufgrund ihrer Mitbestimmungsrechte und angesichts eingespielter Kooperationsbeziehungen in der Regel eine fest etablierte Rolle einnehmen. Bei der Auswahl demografiebezogener Politikkonzepte sind Betriebsräte für das Management ein wichtiges „Ohr" in die Belegschaft hinein. Personalpolitische Themen wie Bedarfsplanung, Rekrutierung oder Aus- und Weiterbildung stehen ohnehin sehr weit oben auf der Agenda der betrieblichen Interessenvertretung. Arbeitspolitische Themenstellungen, die sich zudem auch innerhalb der Betriebe zwischen Tätigkeitsbereichen und Beschäftigtengruppen sehr stark ausdifferenzieren, kommen hierdurch jedoch zu wenig in den Blick. Jenseits der Begleitung von Managementinitiativen, die in vielen Fällen vor allem auf Themen wie Gesundheitsförderung, Wissenstransfer oder Nachfolgeplanung fokussieren, stellen der demografische Wandel sowie Gestaltungsüberlegungen einer alter(n)sgerechten Arbeitspolitik vielfach bisher kein strategisches Thema für die Interessenvertretungen dar. Gleichwohl bieten die in den Betrieben zunehmenden Diskussionen über die Auswirkungen des demografischen Wandels auch einen Hebel zur Thematisierung von arbeitspolitischen Problemen und können helfen, neue Gestaltungsmöglichkeiten zu eröffnen. So finden sich in den von uns untersuchten Betrieben ver- schiedene Beispiele, in denen es Betriebsräten unter Verweis auf die sich weiter verändernden Altersstrukturen der Belegschaft und die damit einhergehenden Probleme und Kosten gelungen ist, sich neue Bündnismöglichkeiten zu erschließen und neue Bewegung in die Diskussion von arbeitspolitischen Problemlagen zu bekommen (vgl. Abschnitt 3).

Neben diesen für Betriebsräte eher generell typischen Handlungssituationen und Orientierungen zeigte sich gerade bei den betrieblichen Interessenvertretungen, dass die Handlungsbedingungen der betrieblichen Akteure auch durch branchen- und tätigkeitsspezifische Problemlagen geprägt sind:

(1) Für Betriebsräte der Chemie- und Pharmaindustrie kommt insbesondere der Gestaltung von Schichtarbeit, aber auch den steigenden psychosozialen Belastungen in Folge von Umstrukturierungen und technologischem Wandel, eine hohe Bedeutung zu. Arbeitspolitik im Sinne der Gestaltung von Arbeitsabläufen und Organisationsstrukturen der Arbeit stellt für sie traditionell zwar kein prioritäres Politikfeld dar, die Aufmerksamkeit für Fragen der Arbeitsgestaltung nimmt in dieser Branche aber zu. Das Thema demografischer Wandel kann hierbei ein wichtiger Hebel sein. Ein illustratives Beispiel ist die Vereinbarkeit von Teilzeit- und Schichtarbeit, die in einem von uns untersuchten Chemiebetrieb vom Betriebsrat schon lange allgemein eingefordert und vom Management so lange als nicht realisierbar abgelehnt worden war, bis die Umsetzung altersbezogener Arbeitszeitabsenkungen (Altersteilzeit, Altersfreizeit) entsprechende Regelungen notwendig machte. Eine wichtige Rolle können in diesem Kontext tarifvertragliche Regelungen spielen, die, wie in der Chemischen Industrie, über ihre Thematisierungsfunktion hinaus den Interessenvertretungen die Chance bieten, im Betrieb Aktivitäten zu „erzwingen“. Auch in diesen Fällen hängen die Ausrichtung von Demografiepolitiken und insbesondere der Stellenwert von entsprechenden arbeitspolitischen Maßnahmen jedoch in hohem Maße von den Orientierungen der Betriebsräte und den auf der betrieblichen Ebene sich herausbildenden Akteurskoalitionen ab. Gerade am Fall der Chemieindustrie wird deutlich, dass tarifvertragliche Regelungen allein keine ausreichende Wirkung entfalten, sondern stets der Aufnahme und Umsetzung durch die betrieblichen Akteure bedürfen, deren Bedeutung sie damit noch unterstreichen.

(2) Im Maschinenbau ist es aktiven Interessenvertretungen gelungen, in der betrieblichen Demografiepolitik dadurch größeres Gehör zu finden, dass sie auf die für diese Branche typische strategische Bedeutung des Themas Fachkräftesicherung und die Notwendigkeit des Erhalts von Erfahrungswissen verweisen. Hierdurch haben nicht nur arbeitspolitische Forderungen nach dem Erhalt hoher Qualifikationsniveaus und einer breiten Arbeitseinsatzflexibilität zusätzliches Gewicht bekommen. Insbesondere in den Montagebereichen, in denen in relevantem Umfang 
nach wie vor auch körperlich schwere und gesundheitlich verschleißende Arbeitsabläufe anzutreffen sind, verbinden sich Fragen der Qualifikationsnutzung mit einer erhöhten Aufmerksamkeit für arbeitsorganisatorische Themen und der Notwendigkeit einer gesundheitsförderlichen Arbeitsplatzgestaltung.

(3) Auch wenn sich die Betriebsräte der Automobilindustrie durch ein hohes arbeitspolitisches Wissen und besonders stark professionalisierte Strukturen der Betriebsratsarbeit auszeichnen, stellen sich die Handlungsbedingungen für sie angesichts der gegenwärtig vorherrschenden arbeitspolitischen Leitbilder ungleich schwieriger dar. Einen besonderen Problemschwerpunkt stellt hier der personalintensive Bereich der manuellen Großserienmontage dar. Die Arbeit dort ist generell und tendenziell zunehmend durch hohe Leistungsanforderungen, geringe Handlungsspielräume und repetitive Arbeitsvollzüge (kurzgetaktete Fließ-, insbesondere Bandarbeit) geprägt, was sich unter anderem in hohen Krankenzahlen und einem steigenden Anteil von Beschäftigten mit Einsatzeinschränkungen niederschlägt. Gleichzeitig haben sich durch neue Produktionssysteme und die zunehmende Ausgliederung von Vormontage- und Nebentätigkeiten die Spielräume für belastungsregulierende Maßnahmen verringert, auch wenn mittlerweile ein breites arbeitswissenschaftliches Wissen über die Probleme dieses Arbeitstyps und arbeitspolitische Gestaltungsalternativen besteht (vgl. hierzu jüngst: Frieling et al. 2012). Wichtige Ansatzpunkte, um unter diesen Bedingungen Einfluss auf Arbeitsorganisation und Arbeitsbedingungen zu nehmen, sind für Betriebsräte die frühzeitige Einflussnahme auf Planungsprozesse, systematische Ergonomiearbeit (nicht selten auf der Basis formalisierter Arbeitsplatzaudits) sowie arbeitspolitische Initiativen im Rahmen der Umsetzung von Produktionssystemen. Allerdings ist der Kostendruck im Bereich der Arbeitsgestaltung in der Branche unverändert hoch. Unter der Oberfläche einer hochgradig professionalisierten und mit erheblichen Ressourcen ausgestatteten Interessenvertretungsarbeit lässt sich beim Thema alter(n)sgerechte Arbeitspolitik daher vielfach zugleich ein hohes Maß an Ratlosigkeit beobachten. Eine strategische Rolle spielt das Thema alter(n)sgerechte Arbeitspolitik vor diesem Hintergrund selbst für Betriebsräte in der Automobilindustrie bisher vielfach eher nicht.

\section{Akteurskoalitionen betrieblicher Demografiepolitik}

\subsection{Strukturelle Probleme}

Betrachtet man die unterschiedlichen Akteursperspektiven in ihrem Zusammenspiel, so wird deutlich, dass hierin wichtige Weichenstellungen für die betriebliche Demografiepolitik, insbesondere eine wesentliche strukturelle Blockade für eine alter(n)sgerechte Arbeits- und Organisationsgestaltung, angelegt sind. Die betrieblichen Personalabteilungen gingen aus den Reorganisationsmaßnahmen der vergangenen Jahre oftmals geschwächt hervor und beschränken sich nunmehr stärker auf die Kernfunktionen von Personalarbeit. Ihre Durchsetzungsstärke sowohl gegenüber den Fachbereichen wie gegenüber den Unternehmensleitungen ist begrenzt. Die von ihnen ausgehenden demografiebezogenen Maßnahmen konzentrieren sich oftmals auf das Spektrum klassischer Personalpolitik. Die Gestaltung von Arbeits- und Organisationsstrukturen einer alter(n)sgerechten Arbeitspolitik steht deutlich seltener auf ihrer Agenda. Das Fachbereichsmanagement ist demgegenüber zwar ein zentraler arbeitspolitischer Akteur, ihm fehlt zumeist allerdings die mit Blick auf den demografischen Wandel notwendige langfristige strategische Orientierung. Stattdessen dominieren hier die Anforderungen des Tagesgeschäfts, auch wenn durchaus die Bereitschaft zur Reaktion auf akut auftretende Probleme besteht. Die Betriebsräte schließlich haben sich das Thema demografischer Wandel bisher noch kaum als strategisches Thema zu eigen gemacht und reagieren demografiepolitisch zumeist eher reaktiv. Nur selten gehen von ihnen arbeitspolitische Initiativen aus.

Bereits dieser kurze Blick auf die wichtigsten betrieblichen Akteursgruppen zeigt, dass die unterschiedlichen demografiepolitischen Perspektiven und die Durchsetzungsfähigkeit der einzelnen Akteure ein wichtiges Erklärungsmoment für die spezifische Ausrichtung der betrieblichen Demografiepolitik darstellen. Gerade eine alter(n)sgerechte Arbeitspolitik bleibt in den Betrieben vielfach ohne Priorität und ohne Treiber. Alle drei Akteursgruppen sind zwar mit den Folgen des demografischen Wandels und alter(n)sbedingten Problemen konfrontiert. Die betrieblichen Akteurskonstellationen sind oftmals aber wenig geeignet, eine große Dynamik bei der Entwicklung und Realisierung von Konzepten einer alter(n)sgerechten Arbeitspolitik zu erzeugen, während personalpolitische Politikkonzepte für die Personalbereiche und Interessenvertretungen leichter umsetzbar sind. Welche personal- und arbeitspolitischen Strategien im Betrieb umgesetzt werden können, ist von der Fähigkeit der Akteure abhängig, andere betriebliche Akteure für diese zu gewinnen und mit diesen Koalitionen einzugehen. In den von uns untersuchten Betrieben ließen sich typische Muster beobachten. In der untersten Zeile der Übersicht 1 haben wir die unterschiedlichen demografiepolitischen Ansätze den hierfür typischen Akteuren bzw. Akteurskoalitionen zugeordnet.

\subsection{Potenziale}

Eine traditionell etablierte und institutionell stark verankerte Akteurskoalition in den Betrieben ist die aus Personalmanagement und Interessenvertretung. Betrachtet man die betriebliche Demografiepolitik, spielt diese Koalition in vielen Betrieben auch beim Thema demografischer Wandel eine zentrale Rolle. Im Vordergrund stehen hierbei allerdings Strategien, die entweder auf die Optimierung der 
betrieblichen Altersstruktur oder auf die individuelle Betriebsbindung und auf verhaltensbezogene Prävention zielen. Während das Zusammenwirken dieser beiden Akteursgruppen im ersten Fall bereits mitbestimmungsrechtlich naheliegt, bestehen im zweiten Fall für beide Akteursgruppen demografiepolitische Gestaltungsspielräume, die sie ohne größeren Widerstand anderer betrieblicher Akteure nutzen können. In der betrieblichen Auseinandersetzung mit dem demografischen Wandel hat sich - vielfach mit Rückenwind von Betriebskrankenkassen - so gerade das Feld der Gesundheitsförderung und individuellen Prävention mit großer Dynamik entfaltet.

Für die Konzipierung und Realisierung einer alter(n)sgerechten Arbeitspolitik erweisen sich hingegen Akteurskoalitionen als wichtig, in denen dem Fachbereichsmanagement eine aktive Rolle zukommt. In den von uns untersuchten Betrieben wurden alter(n)sgerechte Politikkonzepte insbesondere von einer Akteurskoalition aus Fachbereichsmanagement und Betriebsrat forciert, die sich nicht selten als Reaktion auf konzeptionelle oder personelle Schwächen der betrieblichen Personalbereiche zusammengefunden hat. Die Schwäche des betrieblichen Personalmanagements muss keineswegs zu einer Blockade arbeitspolitischer Demografiekonzepte führen. Mal ging die Initiative vom Linienmanagement der jeweiligen Fachbereiche aus, die z. B. im Rahmen der Umsetzung von Produktionssystemen oder bei der Entwicklung von fachbereichsspezifischen Reorganisationsmaßnahmen großen Wert auf eine aktive Einbindung und die gestalterische Mitarbeit von Betriebsräten legten. Mitunter gingen proaktiv orientierte Betriebsräte ihrerseits direkt auf das Linienmanagement zu, um mit ihnen Entwicklungsperspektiven ihrer Fachbereiche zu diskutieren und sie für Gestaltungsprojekte zu gewinnen. Im Fall eines großen Maschinenbaubetriebes beispielsweise nutzte das Fertigungsmanagement die konzernweite Umsetzung eines Produktionssystems für das betriebliche Ziel der Senkung von Durchlaufzeiten und entwickelte gemeinsam mit dem Betriebsrat arbeitspolitische Maßnahmen, die auf breitere Arbeitseinsatzkonzepte und die Qualifizierung der Produktionsbeschäftigten setzten. Beide Seiten versprachen sich hiervon nicht nur einen besseren Belastungswechsel und höhere Einsatzflexibilität, sondern sahen aufgrund des erhöhten Erfahrungsaustauschs und Wissenserhalts auch demografiepolitische Vorteile und strebten eine alter(n)sgerechtere Arbeitsgestaltung an. Der Betriebsrat nutzte die Koalition zudem dazu, in einem ergonomisch besonders problembehafteten Montagebereich Optimierungsworkshops unter aktiver Beteiligung der Beschäftigten zu initiieren. Ähnliche Fälle, in denen sich vor dem Hintergrund eines schwachen betrieblichen Personalbereichs arbeitspolitisch ausgerichtete Koalitionen aus Fachbereichsmanagement und Betriebsrat zusammengefunden haben, finden sich in Fallstudienbetrieben aus allen drei Branchen.

Die bisher genannten Akteurskoalitionen sind in aller Regel auf eines der beiden Politikfelder fokussiert. Während Personalbereiche und Betriebsräte vor allem mit dem Ziel einer demografiesensiblen Personalpolitik zusammenfinden, steht für Koalitionen aus Fachbereichsmanagement und Interessenvertretung die Umsetzung einer alter(n)sgerechten Arbeitspolitik im Vordergrund. Betrachtet man die unterschiedlichen Dimensionen, in denen der demografische Wandel auf die Betriebe wirkt, werden indes die breiten Anforderungen deutlich, auf die eine erfolgreiche betriebliche Demografiepolitik reagieren muss.

Als demografiepolitisch besonders wirksam erweisen sich insofern Akteurskoalitionen, die den Personalbereich, das Fachbereichsmanagement und die betriebliche Interessenvertretung umfassen. Fallstudienbetriebe, in denen dies der Fall war, zeichnen sich nicht nur durch eine größere Umsetzungsdynamik von Konzepten einer alter(n)sgerechten Arbeitspolitik aus. Hier findet sich zugleich auch generell eine größere Aufmerksamkeit für die mit dem demografischen Wandel einhergehenden Problemlagen, und die betrieblichen Akteure decken mit ihren Aktivitäten tendenziell die gesamte Spannweite der in Übersicht 1 unterschiedenen Demografiepolitiken ab. In den von uns untersuchten Betrieben haben wir solche Fälle u. a. dort beobachtet, wo es ein vergleichsweise starker Personalbereich geschafft hat, in enger Abstimmung mit engagierten Interessenvertretungen das Management der verschiedenen Fachbereiche frühzeitig aktiv in die Problemanalyse einzubeziehen und so die Entwicklung alter(n)sgerechter Arbeitspolitikkonzepte anzustoßen. Zum Teil handelt es sich hierbei um Peripherie-Betriebe großer Konzerne, in denen sich solche Dreier-Koalitionen mit dem Ziel der Abwehr zentralistisch vorformulierter arbeitspolitischer Leitideen bzw. zur Durchsetzung standortspezifischer arbeitspolitischer Konzepte zusammengefunden haben. In einem Fall aus der Automobilindustrie ist es auf der Basis einer solchen Akteurskoalition gelungen, bei der Neuplanung für eine zukünftige Produktgeneration an einem bereits bei der Vorgängerfertigung realisierten bandentkoppelten Montagesystem festzuhalten, das von den arbeitspolitischen Akteuren auf der Konzernebene eigentlich nicht gewollt war. Dieses hatte sich aufgrund seiner produktionstechnischen Flexibilität angesichts einer großen Variantenvielfalt aus Sicht des Betriebes jedoch nicht nur aus Produktionssicht bewährt, sondern bot auch in arbeitspolitischer Hinsicht eine Reihe von Vorteilen: Die größere Aufgabenvielfalt, der flexible Arbeitseinsatz, höhere Handlungsspielräume für die Beschäftigten sowie die ergonomischen Vorteile dieses Systems erschienen den Verantwortlichen vor Ort auch mit Blick auf die Erfordernisse des demografischen Wandels als vorteilhaft.

\section{Schlussfolgerungen}

Ausgangspunkt des vorliegenden Beitrags waren der Betrieb als ein zentraler Ort, an dem sich das Altern in der Arbeitsgesellschaft vollzieht, und die Frage nach Gründen für 
die zu beobachtenden betrieblichen Umsetzungsdefizite und -unterschiede demografiebezogener Maßnahmen, wie sie insbesondere für den Bereich einer alters- und alternsgerechten Arbeitsgestaltung zu konstatieren sind. Unseren Untersuchungsergebnissen zufolge liegt in den betrieblichen Akteurskonstellationen ein wichtiger Erklärungsfaktor für die erhebliche Diskrepanz zwischen dem vielfach artikulierten demografiepolitischen Handlungsbedarf der Betriebe und dem gesellschaftlich verfügbaren Wissensstand einerseits und der nach wie vor unzureichenden Umsetzung demografiebezogener Maßnahmen andererseits. Abschließend sollen aus einer solchen analytischen Perspektive heraus ein paar knappe Gestaltungshinweise formuliert werden.

(1) Der in einer ganzen Reihe von Unternehmen zu beobachtende geringe Einfluss des Personalmanagements auf Unternehmensstrategien und der häufig zu beobachtende Kompetenz- und Ressourcenverlust der betrieblichen Personalbereiche erscheint uns als ein gravierendes Problem. Hiermit verlieren die Unternehmen in einem für sie wesentlichen Gestaltungsfeld an Strategie- und Handlungsfähigkeit. Auch aus Sicht der betrieblichen Interessenvertretungen sowie der Gewerkschaften spricht insofern viel dafür, der organisatorischen Stärkung des Personalmanagements mehr Aufmerksamkeit zu widmen.

(2) Der aktiven Einbindung des Linien- bzw. Fachbereichsmanagements in demografiepolitische Maßnahmen kommt eine zentrale Rolle zu. Betriebliche Maßnahmen dürfen hierbei jedoch nicht bei einer Sensibilisierung für Probleme des demografischen Wandels, wie sie gegenwärtig häufig im Rahmen von Führungskräfteschulungen erfolgt, stehen bleiben. Entscheidend ist vielmehr, dass das Fachbereichsmanagement in konkrete arbeitsbezogene Gestaltungsprojekte involviert wird. Dabei mangelt es nicht an möglichen arbeitspolitischen Ansatzpunkten. Aufgrund seiner Handlungsbedingungen dürfte das Fachbereichsmanagement allerdings nur in Ausnahmefällen selbst die Initiative ergreifen.

(3) Wichtig ist weiterhin, dass die Problemlagen im Gefolge des demografischen Wandels in hohem Maß betriebs- und tätigkeitsspezifisch sind. Die Entwicklung von Gestaltungsmaßnahmen sollte daher konkret vor Ort sowie in enger Zusammenarbeit mit den Beschäftigten erfolgen. Dies unterstreicht noch einmal zusätzlich die Bedeutung einer aktiven Rolle des Fachbereichsmanagements und des betrieblichen Personalmanagements und die Notwendigkeit, nicht nur zentral Programme und Tools zu entwickeln, sondern zugleich auf der betrieblichen Ebene über ausreichende Gestaltungsressourcen zu verfügen. Der betrieblichen Interessenvertretung kommt sowohl bei der Einbindung der Beschäftigten als auch gegenüber dem Management bei der Formierung von Akteurskoalitionen eine wichtige Mittlerfunktion bei der Ausarbeitung demografiebezogener Maßnahmen zu.

(4) Und schließlich erscheint es uns wichtig, auf die Anforderungen im Bereich der Arbeitsformen und Arbeitsstrukturen insbesondere der betrieblichen Interessenvertretung hinzuweisen. Während es mit Blick auf die betrieblichen Personalbereiche vor allem um die Bereitstellung ausreichender personeller Ressourcen, Kompetenzen und Qualifikationen geht, die eine lokale Konzept- und Strategieentwicklung ermöglichen, stehen die betrieblichen Interessenvertretungen vor der Frage, wie sie die Bearbeitung eines Querschnittsthemas wie demografischer Wandel in die bestehenden Arbeitsformen integrieren. Eine eindeutige Lösung zeichnet sich hier ge- genwärtig nicht ab. Temporäre Projektarbeitsstrukturen allein dürften aufgrund der Komplexität sowie der politischen Bedeutung des Themas und angesichts der Notwendigkeit, Maßnahmen langfristig zu planen und tragfähige Koalitionen mit anderen Akteuren zu bilden, nicht ausreichen. Denkbar wäre die Bildung eines eigenständigen Arbeitsgremiums zum Thema demografischer Wandel bzw. alter(n)sgerechte Arbeitspolitik. Dieses müsste dann jedoch personell und hinsichtlich seiner Arbeitsweise eng mit den bereits bestehenden Gremien verwoben sein.

\section{LITERATUR}

Becker, F. G./Bobrichtchev, R./Henseler, N. (2006): Ältere Arbeitnehmer und al ternde Belegschaften. Eine empirische Studie bei den 100 größten deutschen Unternehmungen, in: Zeitschrift für Management 1 (1), S. 70-89

Bellmann, L./Leber, U. (2011): Betriebliche Weiterbildung Älterer als Strategie zur Sicherung des Fachkräftebedarfs, in: Sozialer Fortschritt 60 (8), S. 168-176 Bellmann, L./Leber, U./Stegmaier, J. (2007): Betriebliche Personalpolitik und Weiterbildungsengagement gegenüber älteren Beschäftigten. Ein Überblick mit den Daten des IAB-Betriebspanels, in: Loebenstein, H./Schöpf, N. (Hrsg.): Demografischer Wandel und Weiterbildung. Strategien einer alterssensiblen Personalpolitik, Bielefeld, S. $81-97$

Bertelsmann Stiftung/Bundesvereinigung der deutschen Arbeitgeberverbände (BDA) (Hrsg.) (2008): Demographiebewusstes Personalmanagement: Strategien und Beispiele für die betriebliche Praxis, Gütersloh

Betriebskrankenkassen (BKK) Bundesverband (Hrsg.) (2007): Beschäftigungsfähigkeit erhalten! Strategien und Instrumente für ein langes gesundes Arbeitsleben, Essen

Boden, M. (Hrsg.) (2005): Handbuch Personal, Landsberg am Lech

Bögel, J./Frerichs, F. (2011): Betriebliches Alters- und Alternsmanagement. Handlungsfelder, Maßnahmen und Gestaltungsanforderungen, Norderstedt

Buck, H./Kistler, E./Mendius, H. G. (2002): Demographischer Wandel in der Arbeitswelt. Chancen für eine innovative Arbeitsgestaltung, Stuttgart

Bundesministerium für Arbeit und Soziales (BMAS) (Hrsg.) (2010): Aufbruch in die altersgerechte Arbeitswelt, Berlin

Bundesministerium für Bildung und Forschung (BMBF) (Hrsg.) (2010): Demografischer Wandel - (k)ein Problem! Werkzeuge für Praktiker - von Betrieben für Betriebe, Bonn

Deutsche Gesellschaft für Personalführung (DGFP) (Hrsg.) (2011): DGFP-Studie: Megatrends und HRTrends, PraxisPapier 7/2011, Düsseldorf Deutscher Bundestag (Hrsg.) (2002): Schlussbericht der Enquête-Kommission "Demographischer Wandel - Herausforderungen unserer älter werdenden Gesellschaft an den Einzelnen und die Politik", Berlin

Freidank, J./Grabbe, J./Kädtler, J./Tullius, K. (2011): Altersdifferenzierte und altersgerechte Betriebs- und Tarifpolitik. Eine Bestandsaufnahme betriebspolitischer und tarifvertraglicher Maßnahmen zur Sicherung der Beschäftigungsfähigkeit, Dortmund

Frerichs, F. (1998): Älterwerden im Betrieb. Beschäftigungschancen und -risiken im demographischen Wandel, Opladen

Frieling, E./Kotzab, A./Enriquez-Diaz, A. (2012): Mit derTaktzeit am Ende: Ältere Beschäftigte in der Automobilmontage, Stuttgart

Georg, A./Barkholdt, C./Frerichs, F. (2005): Modelle alternsgerechter Arbeit aus Kleinbetrieben und ihre Nutzungsmöglichkeiten, Dortmund

Grewer, H. G./Matthäi, I./Reindl, J. (2007): Der innovative Ältere. Warum die Entwickleruhr länger als sieben Jahre tickt, München/Mering Institut für angewandte Arbeitwissenschaft (IfaA) (Hrsg.) (2009): Der demografiefeste Betrieb, Köln

IG Metall (Hrsg.) (2012a): Alternsgerechtes Arbeiten und demografischer Interessenausgleich, Frankfurt a. M.

IG Metall (Hrsg.) (2012b): Betriebsrätebefragung "Gute Arbeit - gut in Rente" (Pressekonferenz: Handout Befragungsergebnisse), http://www.igmetall.de/ cps/rde/xbcr/SID-0270AEA3-4139610A/internet/docs_ig_metall_

xcms_190162_190163_2.pdf (letzter Zugriff: 22.11.2012)

Kistler, E. (2008): „Alternsgerechte Erwerbsarbeit”. Ein Überblick über den Stand von Wissenschaft und Praxis, Düsseldorf 
Kistler, E./Ebert, A./Guggemos, P./Lehner, M./Buck, H./Schletz, A. (2006): Altersgerechte Arbeitsbedinungen. Machbarkeitsstudie (Sachverständigengutachten) für die Bundesanstalt für Arbeitsschutz und Arbeitsmedizin, Dortmund Kocka, J./Staudinger, U. (Hrsg.) (2009): Gewonnene Jahre. Empfehlungen der Akademiengruppe Altern in Deutschland, Stuttgart

Latniak, E./Voss-Dahm, D./Elsholz, U./Gottwald, M./Gerisch, S. (2010): Umsetzung demografiefester Personalpolitik in der Chemischen Industrie: Inhaltliche und prozessuale Analyse betrieblicher Vorgehensweisen, Düsseldorf

Morschhäuser, M. (2006): Reife Leistung. Personal- und Qualifizierungspolitik für die künftige Altersstruktur, Berlin

Pack, J. (2002): Herausforderungen der betrieblichen Personalpolitik durch alternde Belegschaften, in: Götz, K. (Hrsg.): Personalarbeit der Zukunft, München/Mehring, S. 127-140

Rothkirch, C. von (Hrsg.) (2000): Altern und Arbeit: Herausforderung für Wirtschaft und Gesellschaft, Berlin

Towers Watson (2011): Der demografische Wandel. Auswirkungen für Unternehmen in Deutschland, o. $\mathrm{O}$.

\section{AUTOREN}

KLAUS-PETER BUSS, Dr. des., Wissenschaftler am Soziologischen Forschungsinstitut (SOFI) der Universität Göttingen. Arbeitsschwerpunkte: Arbeits- und Industriesoziologie, berufliche Bildung, Wissen und Innovation.

klaus-peter.buss@sofi.uni-goettingen.de

MARTIN KUHLMANN, Dr., Wissenschaftler am SOFI. Arbeitsschwerpunkte: Arbeits- und Industriesoziologie, industrielle Beziehungen.

martin.kuhlmann@sofi.uni-goettingen.de 